\title{
DEFINITION OF NON-LETHAL WEAPON IN INTERNATIONAL
}

\section{LAW}

\author{
Gennadiy Kurdyukov ${ }^{1}$ \\ Anastasia Drygina $^{2}$
}

\begin{abstract}
This paper examines the terminology, reveals the definition of non-lethal weapon, provides a detailed analysis of the criteria for lethality of weapons, distinguishes between the concepts of non-lethal and lethal weapons, and non-lethal weapons are also separated from other items used as weapons. The international legal science also lacks the universally accepted definition of the non-lethal weapon concept. That is why the authors have paid attention to the question of terminology in relation to this weapon, as well as to the criteria on the basis of which it can be distinguished from other types.
\end{abstract}

Keywords: non-lethal weapons, conventional weapons

\section{Introduction}

The definition of non-lethal weapons is a cornerstone in the study of this topic. The term non-lethal weapons is absent in international documents, and that is not surprising since phenomenon as non-lethal weapons was considered only in the 1960s. At this point, perhaps the most famous of all the definitions are given by directive No.3000.3 of the United States [Susan, Joseph and Rutigliano, 2015]. Non-lethal weapons are designed and used to minimize the possibility of causing death, excessive (serious) and permanent damage [Stephen, 2015].

\section{Methods}

In the process of research, the authors used general and special methods of scientific knowledge. The main research methods used in writing this paper were the dialectical method, the method of generalization, complex and system analysis, synthesis, etc. Special methods were also used: formal

\footnotetext{
${ }^{1}$ Faculty of Law, Kazan Federal University, Kazan, Russia

${ }^{2}$ Kazan Federal University. e-mail: ana_drygina@mail.ru
} 
legal, comparative legal, logical, historical, etc.

\section{Results and discussion}

The term non-lethal weapons is very controversial since the unlimited scope of hard law provides authors who study the nature of these weapons with complete freedom in choosing name.

Thus, the following terms can be used to define non-lethal weapons:

Disabling/neutralizing weapons (soft-kill weapon);

- Sub-lethal / close to a mortal weapon etc. [David, 1999]

The versions sounded above show that even in the original language the term does not sound very successful (for example, less-than-lethal is literally as less than deadly. This definition raises many questions: what is a deadly weapon? To what extent does a nonlethal weapon differ from a deadly weapon etc.

To define the concept of a weapon that does not cause serious damage to human life and health, the authors generally still use the term nonlethal weapon. the term non-lethal weapons is used to describe weapons, the damage from which has a temporary, reversible effect, less compared to using other types of weapons.

Some experts consider the term oxymoron. Others believe that this name is misleading people, as these weapons can cause damage, and, accordingly, this damage can be fatal. The third, and, in our opinion, the most logical point of view is that the term non-lethal is contrasted with the notion of conventional (lethal), which means its main characteristic is emphasized: this weapon was not created to destroy or kill, but in order to disable the enemy [Bryan, 2010].

However, all these definitions have a similar problem: a weapon, by its very nature, leaves the possibility of a lethal outcome, even if it is theoretical.

\section{Summary}

To isolate this weapon as a specific type of weapon, it is necessary to draw a distinction between it and other weapons. In our opinion, such a distinction should be made in two categories:

1. First, we need to find out what are the differences between nonlethal weapons and lethal weapons. 
In different situations, the same weapon can be used in completely different ways and, accordingly, its use may have different consequences. In one case, it will not cause any harm to a person, and in another situation, it can take life. Therefore, it seems to us that the distinction between non-lethal and lethal weapons should be made according to the purposes and methods of their use.

As for the purpose of using weapons, there are international treaties (conventions) that restrict and prohibit a specific type of weapon. Such weapons are called conventional. The purpose of these documents is not just to prohibit/restrict the use of weapons, but to exclude the most undesirable results of their use: the inevitability of death, excessive damage and unnecessary suffering, damage to protected categories of people. However, international treaties prohibiting or limiting weapons do not exclude the possibility of death in principle, they only try to regulate that death will not be inevitable, and the damage caused by the weapon will not cause unnecessary suffering.
However, the use of non-lethal weapons does not exclude the negative consequences. It can also be indiscriminate, and cause unnecessary suffering; it is enough to cite the statistics of victims during the operation to liberate the theater complex in Dubrovka (Moscow) in 2002 (Nord-Ost) (despite the fact that the European Court of Human Rights.

Between the objectives of using both types of weapons, there is a very fine line, which really can be found only at the time of planning the use of weapons. If, in the case of the use of a lethal weapon, the death of the enemy is undesirable, but this is the normal state of affairs and avoiding death by a person during a battle is a positively perceived fact, but rather an exception to the rule, in the case of using non-lethal weapons, on the contrary, it is individual's death will be an exception, some kind of system failure. This is an abnormal and unexpected result of the use of such weapons, and this may mean that they are used either incorrectly or not in accordance with their intended purpose, and therefore, most likely, illegally.

The second important criterion for distinguishing is the method of using 
weapons. Here, much depends on the circumstances of the use of weapons, the reasons, the intentions of those who use weapons, etc.

Thus, a striking example of the improper use of weapons is the storming of a theatrical complex in Dubrovka during an operation to free hostages. After three days of the siege of the theatre, October 26, at about $5.00-5.30$, sleeping gas began to penetrate through the ventilation.

However, the circumstances were such that:

- First, the actions of the numerous rescue services which were on the scene of the terrorist act were not coordinated. The operation involved the Center for Emergency Medicine, the doctors of the ambulance brigades, the doctors of the city hospitals, the rescue service, and each unit had its own management layer, communications, activity protocols, etc. The result of the lack of interaction was that when providing help, doctors did not know what type of treatment the victims had received, and perhaps some of the victims received injections in double size, and some did not receive them at all.
- Secondly, the medical staff was not warned that a chemical substance would be used during the operation. The doctors were preparing to work at the site of the explosion and, therefore, the rescue teams mainly consisted of surgeons, not toxicologists. Moreover, assisting the victims, the doctors did not know what kind of substance was applied to them).

- Thirdly, the mass evacuation of hostages was difficult. Heavy trucks and bulldozers placed nearby prevented the normal movement of ambulances etc.

A similar situation occurred during the hostage-taking in Peru in 1997. However, the Peruvian authorities sought the opinion of American experts on the safety and feasibility of using phytanyl in the assault. Since it turned out to be impossible to organize mass medical care, the Peruvian authorities decided not to use the gas.

Another example is the use of stun guns and Taser by police officers towards detainees. For example, from 2001 to 2012 in the United States, about 500 people died in places of detention and custody, being attacked by Taser. However, inadequate use of Taser by police does not make them potentially 
dangerous weapons requiring immediate restrictions controlled by international law.

Any use of force, regardless of whether it was carried out with the help of lethal or non-lethal weapons, must be justified by a certain level of damage that followed after the use of weapons. Pepper sprays and shock batons are not always automatically applied just because they are classified as non-lethal weapons. Instead of relying on broad characteristics, we must evaluate the nature of the situation to which force must be applied.

Is it possible to say that a weapon that was initially used as a nonlethal one changes its status in the application process depending on the circumstances and the method it was applied? We tend to think that the first criterion - the purpose - is the main one and determines the second one. In the case when the use of weapons entails victims and serious losses, this does not mean that at this moment the weapon changes its status. This means that in such a situation there is incorrect or illegal use of non-lethal weapons.

2. In what aspects non-lethal weapons differ from items that are not weapons, but which can be used as weapons in certain circumstances?

During the period of work on this topic, the authors periodically were asked the question: what is the difference between non-lethal weapons and objects that, while not being such by their very nature, can nevertheless serve as weapons?

Moreover, there is a point of view among experts that the devices described above are not a weapon. Proponents of this idea call them special non-lethal agents and believe that they do not belong to the weapon, arguing that in this case two incompatible concepts are combined. In support of this position, one can cite the fact that a weapon, unlike the special agents, is subject to registration; also there is different responsibility for the possession and use of weapons and special equipment (criminal and administrative, respectively); in most countries, possession, carrying and use of special agents (such as a traumatic gun, aerosol cans with irritant means, and electroshock devices) for personal protection is not prohibited etc. 
However, we incline to believe that non-lethal weapons as a type of weapons do exist and require regulation.

First, there is the opinion of the ICRC on this issue. According to Article 36 of Additional Protocol I of 1977, if there are doubts whether a device or a system is a weapon, it is necessary to seek advice from the authority that carries out the weapon review. Such body is usually a committee established on the basis of the Ministry of Defense or another similar institution. It includes lawyers, military personnel, as well as representatives of other departments (military doctors, specialists responsible for planning and logistics, military engineers) and external experts.

Secondly, in the national legislation of most countries, there are certain lists of weapons. For example, in the Russian legislation, there is Weapons Act which contains the definition of the concept of weapons, the Order of the Government of the Russian Federation dated August 3, 1996, N 1207-p List of service and civilian weapons and ammunition and civilian weapons. There is no specific notion of non-lethal weapon, but, on the other hand, it does not say that the list is closed.
Thirdly, in the works of specialists, there are often approximate lists of weapons that are constantly used by the authors in compiling classifications. Such lists are in the works of S. Casey-Maslen, S. Wright and others. So, in many studies there is a similar thesis that there are chemical non-lethal (sleeping gas), biological (defoliants, herbicides), acoustic (sound cannons and special acoustic devices) etc. The works of eminent scientists are an optional source of international law in accordance with the statute of the International Court of Justice. Accordingly, a study with a list of weapons established in it may subsequently receive a doctrinal expression.

\section{Conclusions}

So, despite the fact that the status, rules of application and even the definition of the concept of non-lethal weapons are grey area of international law, this phenomenon exists in our life. In each specific situation, non-lethal weapons show themselves in different ways, which is why it is not yet possible to form a definite opinion about them. However, undoubtedly, the use of non- 
lethal weapons is an interesting and urgent issue that will force the scientific world to talk about it many times.

\section{References}

Susan D. LeVine, Joseph A. Rutigliano Jr., (2015). U.S. Military Use of Non-

Lethal Weapons: Reality vs Perceptions, 47 Case Western Reserve Journal of International Law, p. 239, 2015. URL: http://scholarlycommons.law.case.edu/ji 1/vol47/iss1/18239

Stephen Coleman, (2015). Possible Ethical Problems with Military Use of Non-Lethal Weapons, Case Western Reserve Journal of International Law , 47, p. 186-187, 2015.

David P. Fidler, (1999). The International Legal Implications of Non-Lethal Weapons, Michigan Journal of International Law, Articles by Maurer Faculty, Paper 699, p. 54, 1999.

Bryan v. McPherson, (2010). US Court of Appeals for the Ninth Circuit. 630 F.3d 805; 2010 Daily Journal D.A.R. 17.910 